\title{
THERMAL ANALYSIS OF CPU WITH VARIABLE BASEPLATE HEAT- SINK USING CFD
}

\author{
Channamallikarjun \\ Department of Mechanical Engineering, BKIT-Bhalki-585328
}

\begin{abstract}
The computational fluid dynamics is concentrated on the forced air cooling of the CPU using a heat sink. This paper utilizes CFD to identify a cooling solution for a desktop computer, which uses an $80 \mathrm{~W}$ CPU maximum whereas this number will be increased in the range of 70-120W in the forthcoming desktop computer systems. This paper considers the optimal plate fin heat sink design and cylindrical fin heat sink design with variable copper base plate and the control of CPU heat sink processes. To have a better heat dispersion performance, a computational fluid dynamics is utilized to search for an optimal set of plate-fin. Base plate thickness, fin thickness, fin profile and fin material parameters are to be handled together due to the frequently encountered space limitations. The different heat sink designs are analyzed by using commercial CFD software packages ANSYS design modeler for modeling and ANSYS meshing for CFD meshing and finally ANSYS fluent is used for CFD solver. The well converged; results are compared with the experimental data. Especially, replacing aluminum with copper as base plate material improved the performance. This study will benefit the design engineers involved in electronic cooling and also help to reduce the significant increases in the sound power emitted by the CPU.
\end{abstract}

\section{INTRODUCTION}

Due to cost constraint, predictable air cooling technology with a fan, heat sink grouping broadly used to cool desktop computers as shown in fig 1 .

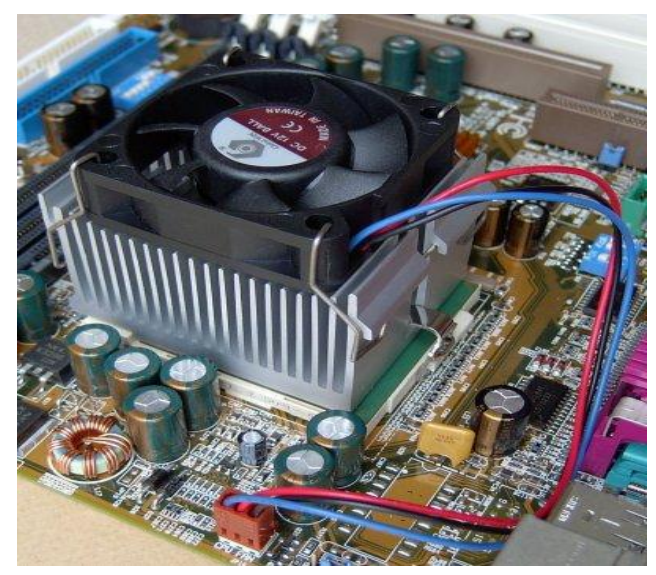

Fig1.Computer heat sink mounted onto a motherboard

Computer cooling is necessary to take away the dissipate heat formed by computer components, to stay components contained by their safe working temperature restrictions. different cooling methods help to get better processor presentation or reduce the sound of cooling fans.

Components which produce heat and are susceptible to performance loss and damage include integrated circuits such as CPUs, chipset and graphics cards, along with hard drives.
Over heated parts fail early. There is a need to study the heat dissipation in the computer for different condition. Hence CFD analysis of heat dissipation is carried out in this project and presented.

\subsection{Organization of the Report}

Chapter 1 includes general introduction to heat sink and organization of the report.

Chapter 2 includes literature review in which working principle and about the software, objective, statement and scope of the project is defined.

Chapter 3 includes the methodology in which how the model is created is discussed and model meshing is done by ANSYS and boundary conditions are well defined.

Chapter 4 includes the result and discussion.

Chapter 5 gives the conclusion of the report.

\section{LITERATURE SURVEY}

\subsection{Introduction}

This chapter basically covers the working principle involved. After that it focuses on the literature review that has been carried out for this work. Based on detailed study of literature review objective of the project has been explained and statement of the project work is defined. In last chapter scope of the project work has been explained.

Heat sink: The performance criterion of heat sinks is the thermal resistance, which is expressed as the temperature difference between the electronic components and ambient per 
watts of heat load. It is expressed with units $\mathrm{K} / \mathrm{W}$. Today's electronic chips dissipate approximately $70 \mathrm{~W}$ maximum whereas this number will be multiples in the near future. The temperature differences from the heat sink surface to the ambient range from $10{ }^{\circ} \mathrm{C}$ to $35^{\circ} \mathrm{C}$ according to the heat removal capability of the installed heat sink. Heat sinks may be categorized into five main groups according to the cooling mechanism employed [4]:

System constraints: During the design period of a heat sink the system constraints are to be determined first. System constraints are parameters that are out of control of the designer.

Heat to be removed: The most important system constraint is the rate of heat to be removed. It is generally assumed to be a fixed value, which is in fact the maximum heat dissipation rate of the electronic component even if the heat dissipation has a transient manner. The dissipated heat is due to the inefficiency of the electronic component and it is the difference between the input and output.

Electrical power: The maximum operating temperature: It is generally determined by the material properties of the electronic component. Most components which are not specifically designed for military applications have a maximum operating temperature which is less than $100{ }^{\circ} \mathrm{C}$. Allowable thermal resistance is calculated. by using the dissipated power and the temperature difference between the maximum operating temperature and the ambient temperature. A computer uses a CPU with $40 \mathrm{~W}$ heat dissipation rate and $80^{\circ} \mathrm{C}$ of maximum operating temperature in an ambient of 30 ${ }^{\circ} \mathrm{C}$, needs a heat sink with thermal resistance of $1.25 \mathrm{~K} / \mathrm{W}$ or lower. This number should include the thermal resistance of the thermal interface material used to bond the heat source and heat sink and also chip's own internal thermal resistance from its junction to the chassis.

Ambient temperature: Since the air blown on the heat sink by the fan is at the temperature of the ambient, the thermal resistance is calculated by using the ambient temperature. For closed domains such as a computer chassis, the air inside the chassis will be hotter than the outside air, so average temperature of inside air instead of ambient air temperature must be used for thermal resistance calculations.

The maximum volume of the heat sink: This is another constraint when limited space around circuit boards is considered. For forced convection applications, the size of the fan should also be considered. A typical heat sink, Alpha PAL $8045 \mathrm{~A}$, occupies a total volume of $80 \times 80 \times 84 \mathrm{~mm}^{3}$, including the fan. For applications where the electronic box does not have enough space, heat pipes may be used to carry the heat load to a location where more space is available to use a big heat sink.
Sealing: In some cases the necessity for sealing out the dust and sand creates another system constraint. It prevents the use of external fans, as a result, cooling mechanisms are limited to radiation and natural convection through external surfaces. If a fan is to be used, blowing fans are preferred since they increase the static pressure inside the chassis. Air filter is considered for sucking fans especially when the components are sensible to dust.

\subsection{Design Parameters}

Once the system constraints are determined, design parameters are to be considered. The design parameters include the heat sink material, the number and geometry of the fins and their alignment and the base plate thickness. In order to obtain the minimum thermal resistance and pressure drop, each of these parameters must be designed well.

Material: Heat sink materials are generally etched silicon for small dimensions and metals for larger sizes. Metals with high thermal conductivity and relatively low cost are preferred, like aluminum and copper. Combinations of different materials are possible like the use of aluminum fins bonded to a copper base as in as shown in fig. 2. Although the thermal conductivity of zinc is lower compared to that of aluminum and copper, it may also be a good material for electronic cooling purposes. When zinc added to an alloy, [6]it eliminates porosity in the casting process, which is an advantage over aluminum and copper since they are not pore free after the casting.

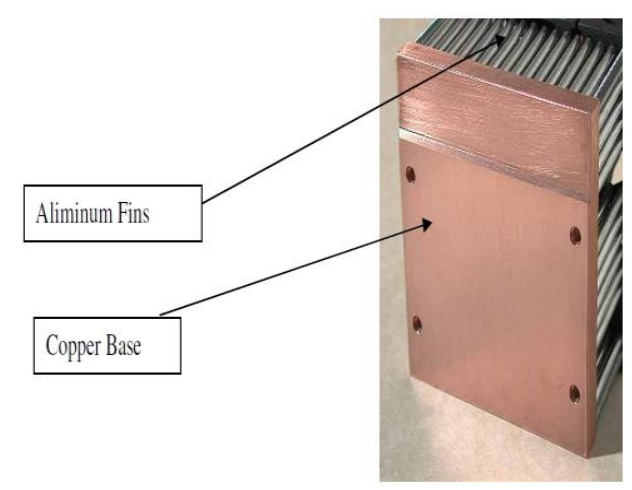

Fig.2. Heat Sink Material

The number of the fins: It is one of the most important factors for heat sink performance. A heat sink designed for electronics cooling is a compact heat exchanger for which the ratio of heat transfer area to occupied volume is very large. The heat transfer area is enhanced by use of fins. Therefore increasing the number of fins provides more area for heat transfer. Increasing the number of fins from 238 to 294, Hedgehog increased the heat transfer area by $8.4 \%$ and approximately 10 $\%$ efficiency is assured in the succession of Hedgehog-238M 
to Hedgehog-294M [7]. However, it should be noted that increasing the number of fins creates an adverse effect, which is the increased static pressure drop. In order to overcome higher pressure drops, higher pumping powers are needed, which requires the installation of more powerful fans or blower.

Fin shapes: Different kinds of heat sink geometries are possible. Pin fins, straight fins, fluted fins, wavy fins and fins with non-standard geometry are possible. The most common ones are pin fins whose cross section can be round, square, elliptical, hexagonal or any other suitable geometry. Straight fins that have rectangular cross sections are also widely used. Depending on the spacing among the fins of a heat sink, flow requirements and pressure drops may differ. Design engineers try to achieve the minimum thermal resistance with the pressure drop as low as possible by modifying the fin shapes. Extensive literature is available on this subject. Chapman et al. [5] showed that for the horizontal flow case using elliptical pin fins instead of rectangular ones reduces the vortex shedding and minimizes the pressure drop. Consequently, the thermal resistance is reduced due to the lower temperatures which is a result of larger heat transfer area exposed to the flow. Fluted and wavy heat sinks are not commonly used due to performance and manufacturability problems. Sikkaet al. [8] showed such heat sinks did not yield significantly better thermal resistance than the conventional heat sinks for natural and low velocity forced convection.

Sathyamurthy [9] demonstrated that when the fins of a rectangular cross section fin heat sink are divided into pieces in the flow direction and forming a staggered alignment, the thermal performance is enhanced by $8 \%$ at the expense of 10 $\%$ to $20 \%$ pressure drop. [12] The reason is that a staggered fin heat sink enhances heat removal by repeated interruption and re-initiation of the boundary layer on the fin surfaces. Understanding this phenomenon, the design engineers prefer to install pin fin or divided fin heat sinks instead of planar fin heat sinks. Fin alignment: Alignment of the fins on the base plate plays an important role especially for the heat sinks which are cooled by fans installed by their side.[11] We only considered the heat sinks that are cooled by fans on the top. For side cooled heat sink, fin alignment may be inline or staggered. Although the staggered design is superior to the inline design under the same flow conditions, Dvinskyet al. [8] showed that inline design performs better than staggered for their specific case when the same fan is used. The explanation for this behaviour is the higher flow resistance of the staggered heat sink, which causes more air to bypass the heat sink.

Technically, fins can be manufactured with a height approximately 80 times their thickness or diameter but since the rate of increased performance becomes less as height is increased, fin height of 45 times the thickness is the suggested maximum [9].

\subsection{Objective of the Project}

From the literature review it is observed that removal of heat developed in the CPU of computer causes operational damage and reduces the performance of electronic chips. Also it is observed that heat source in these electronic circuit are discrete in nature. These discrete heat sources in the CPU and their effect on the performance is complicated and combursum analysis. Conducting the experiment of these minute electronics components and their heat dissipations study experiment is a time consuming and costlier. With the use of CFD analysis the heat transfer characteristics can be easily studied hence there is a need for CFD analysis of CPU of computer for its flow characteristics.

\subsection{Statement of the Project}

From the literature review and objective of the project it is decided to perform a CFD analysis of CPU with variable heat sink for fluid flow and heat transfer characteristics.

\subsection{Scope of the project}

The scope of the project is as follows

- Perform CFD simulation of plate heat sink with base plate aluminum compare the effects in terms temperature and pressure drop.

- Perform CFD simulation of plate heat sink with base plate copper compare the effects in terms temperature and pressure drop.

- Perform CFD simulation of circular pin-fin heat sink compare the effects in terms temperature and pressure drop.

- Perform CFD simulation of elliptical pin-fin heat sink compare the effects in terms temperature and pressure drop.

\section{METHODOLOGY}

In this chapter the physical modeling of CPU with its peripheral electronic equipment and its meshing is presented. The boundary condition for the given problem is specified.

\subsection{Modeling}

Geometrical Model: Geometry files are created in ANSYS Design Modeler as shown in fig 3 and imported into ANSYS Meshing to create computational Mesh. 


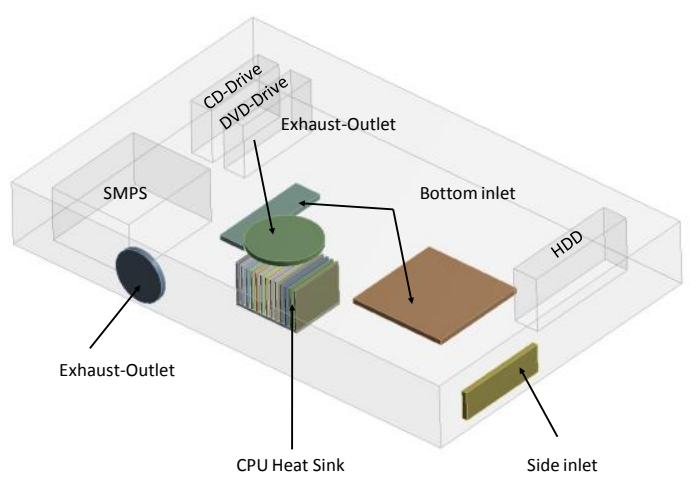

Fig.3 Heat sink geometry plate-heat sink

\subsection{Meshing}

After creating the geometric model using the ANSYS design modeler the CFD meshing of heat sink is carried out in ANSYS meshing software.

In this p Patch-conforming mesh method is used for meshing all the absorber tube and sweep method is used for absorber plate.

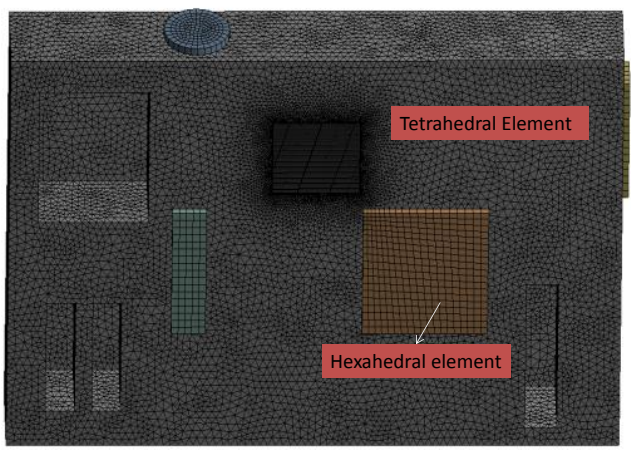

Fig.4 CFD domain meshing of CPU

\subsection{Boundary Conditions}

In this analysis these blocks are modeled and only heat sink is modeled as sold domain with heat source of $80 \mathrm{~W}$. In this case heat sink material considered as aluminum. Heat sink base plate material considered as aluminum as well as copper.

Table 1 Heat sink material and its properties.

\begin{tabular}{|l|l|l|}
\hline Property & Aluminum & Copper \\
\hline Density kg/m3 & 2719 & 8978 \\
\hline $\mathrm{Cp} \mathrm{j/kg-k}$ & 871 & 381 \\
\hline $\begin{array}{l}\text { Thermal conductivity } \\
\text { W/m-K }\end{array}$ & 202.4 & 387.6 \\
\hline
\end{tabular}

The fans inside the domain are modeled as circular surfaces which defined as heat flow rate outlet as shown in the table 2 .

Table 2 Heat flow rate of Fans

\begin{tabular}{|l|l|}
\hline Name of the Fan & heat Flow Rate \\
\hline CPU Heat sink fan & 30 CFM \\
\hline Case Fan & 40 CFM \\
\hline
\end{tabular}

\section{RESULTS AND DISCUSSIONS}

In this chapter the different heat sink design are studied and presented. The temperature contours, pressure contours, and velocity contours of different heat sink design are studied. Solution of the governing equations presented in the previous section was achieved using the commercial code Fluent. This program uses the finite-volume approach whose basic feature is the integration of the governing equations over a control volume to yield the discretized equations. Hence the first step is the subdivision of the computational domain into finite number of control volumes. The method is locally conservative as it is based on a local balance of fluxes in each control volume. In this case used unstructured mesh to solve the heat sink simulation.

\subsection{Results}

\subsubsection{Plate Heat Sink-Aluminum}

The ANSYS FLUENT CFD code was used for the simulations. Fine meshing was used. And at the outlet showing maximum pressure drop which is shown with light blue color.

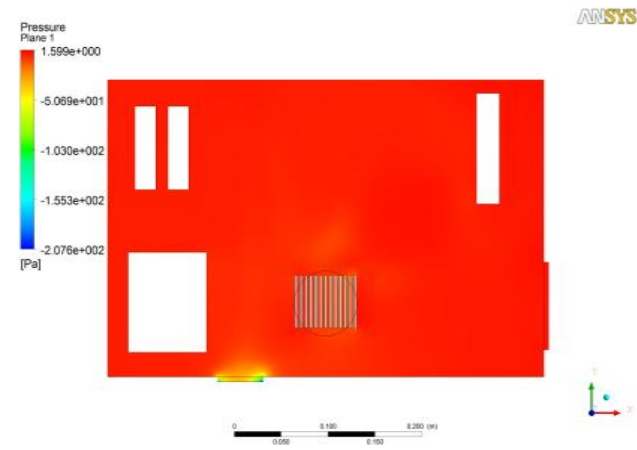

Fig.5.Pressure contours-mid section of CPU 


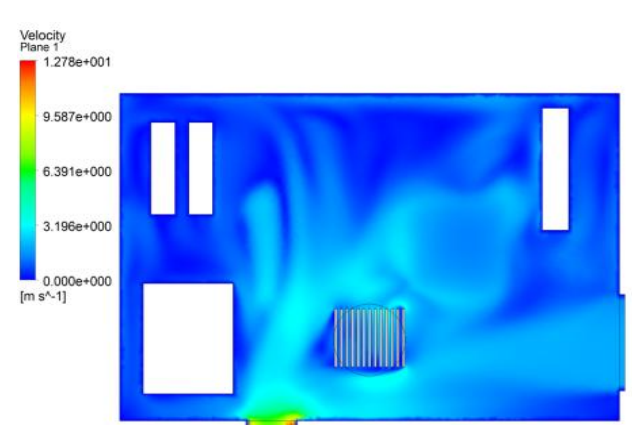

Fig.6 Velocity contours-mid section of CPU

Fig.6 shows the Velocity contours-Mid section of CPU. It shows the velocity distributions in the heat sink region. In some region, the magnitude of the velocity in the bypass region is higher than the inlet velocity.

Figure 7 shows the flow and temperature behavior of the air entering the heat sink and the side. In Fig.4.4 bottom side of the heat sink shows the higher temperature approximately 330 $\mathrm{K}$.

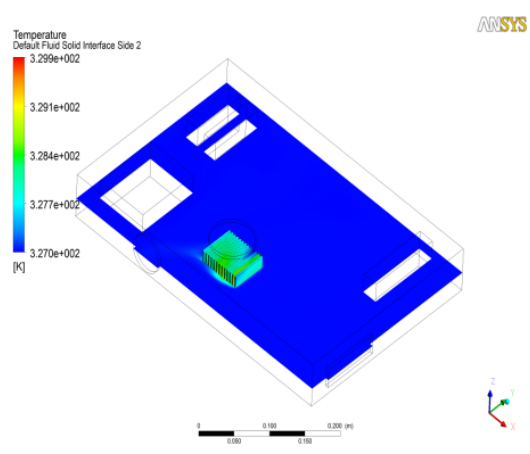

Fig.7.Temperature contours-mid section of CPU

\subsubsection{Plate Heat Sink-Copper Base}

In this section keeping other parameters and only change base plate material with copper is carried out. Copper base plate heat sink performs well when compared to aluminum base plate heat sink. The fig8 shows the pressure distributions of mid-section of CPU and it shows the lesser pressure drop. And at the outlet showing maximum pressure drop which is shown with light blue color. The fig 9 shows the Velocity contoursMid section of CPU. It shows the velocity distributions in the heat sink region. In some region, the magnitude of the velocity in the bypass region is higher than the inlet velocity.

The temperature distributions are as shown in fig 10. In this improving thermal conductivity by using copper instead of aluminum is considered. First, it was seen that copper, the thermal performance of the heat sink was not affected. This also indicates that this copper base heat sink do not contribute much to the heat transfer. The base is made of copper, maximum temperature on the heat sinks decreased by $1{ }^{\circ} \mathrm{C}$. It was also seen that the minimum temperature increased by more than $0.5^{\circ} \mathrm{C}$.

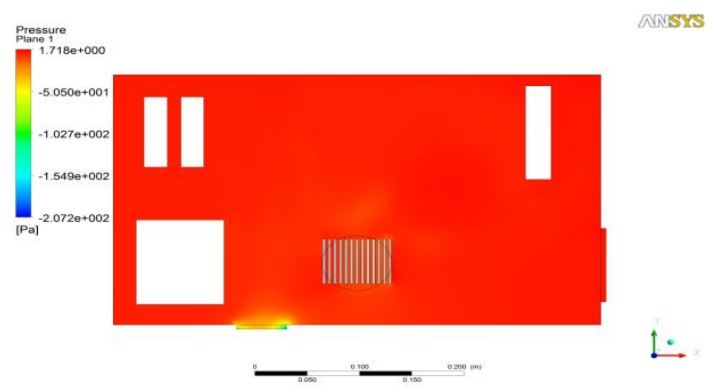

Fig.8 Pressure contours-mid section of CPU

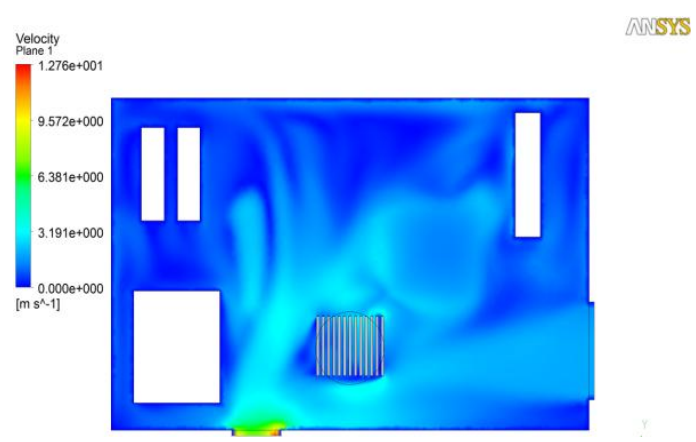

Fig.9 Velocity contours-mid section of CPU

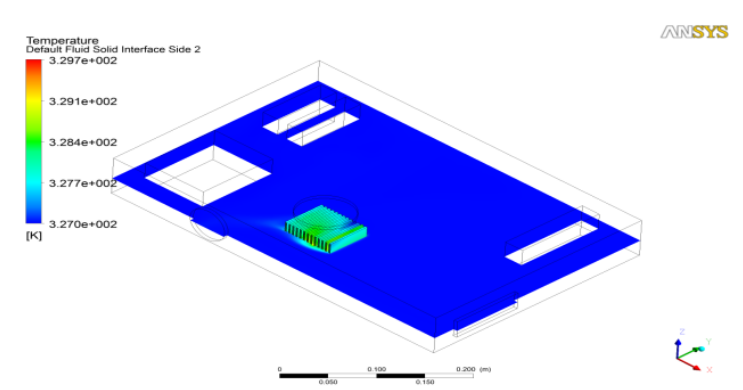

Fig.10.Temperature contours-mid section of CPU

Figure.10 shows the temperature contours at heat sink. The air enters the system at $27^{\circ} \mathrm{C}$ and its temperature increases along the flow path. At the inlet of heat sink fan, it is heated to about $31{ }^{\circ} \mathrm{C}$ and its temperature is further increased to $50{ }^{\circ} \mathrm{C}$ while flowing over the heat sink.

The performance of plate fin heat sink model is better when compared to all cylindrical fin heat sink models. Even though it has less the number of fins it performs well. Copper base plate heat sink performs well when compared to aluminum base plate heat sink. Also the effective heat transfer elliptical and circular decreases in this heat sink compared to plate heat sink. Elliptical and circular heat sink performance can be increased by increasing the number of fins. 
The experimental deviation is higher in Circular heat sink Aluminum base plate because the number of fins is reduced to computational time because the number of elements is close to 2 millions, further increase in number of fins the CPU time will be more and require higher capability system.

Table 3 Temperature for plate heat sinks for $80 \mathrm{~W}$ CPU heat dissipation

\begin{tabular}{|l|l|l|}
\hline $\begin{array}{l}\text { Temperature } \\
\text { Difference K }\end{array}$ & $\begin{array}{l}\text { Plate Heat } \\
\text { Sink } \\
\text { Aluminum } \\
\text { base plate }\end{array}$ & $\begin{array}{l}\text { Plate Heat } \\
\text { Sink copper } \\
\text { base plate }\end{array}$ \\
\hline $\begin{array}{l}\text { CFD } \\
\text { Simulation }\end{array}$ & 26 & 23.4 \\
\hline $\begin{array}{l}\text { Experimental } \\
\text { Work }\end{array}$ & 24.5 & 19.8 \\
\hline
\end{tabular}

\section{CONCLUSIONS}

In this paper, the CPU cooling performances of a computer chassis with rectangular, pin fin heat sinks were investigated using ANSYS Fluent and the results were compared.

The heat sink temperature difference results have been compared with an experimental result to find out best heat sink designs, and it shows the good correlation.

The fin profiles the base plate thickness were investigated for enhancing the heat dissipation rate from CPU, and some thermal improvements as well as space reduction and material savings were attained. Improvements on heat sink designs are possible by the use of CFD. Eventually it is possible to finish up with a new heat sink design which has better thermal performance and uses less material.

\section{REFERENCES}

[1]. Kim Fowler, "Heat Transfer and Cooling," IEEE Instrumentation \& Measurement Magazine, pp 48-51, 2001

[2]. Keller, Kurtis, 1998 "Low Cost, High Performance, High Volume Heatsinks", IEMT-Europe.

[3]. Takeshi hirasawa, Kenya Kawabata and Masaru Oomi, 2005, "Evolution of heat sink technology", Furukawa Review No.27, pp. 25-29

[4]. Seri Lee, "Optimum Design and Selection of Heat Sinks," Eleventh IEEESEMI-THERM Symposium, pp. 48-54, 1995.

[5]. Christopher L. Chapman, Seri Lee, Bill L. Schmidt, "Thermal Performance of an Elliptical Pin Fin Heat Sink," Tenth IEEE SEMI-THERM, pp.24-31, 1993.

[6]. K.K. Sikka, K.E. Torrance, C.U. Scholler and P.I. Salanova, "Heat Sinks with Fluted and Wavy Fins in Natural and Low-Velocity Forced Convection," Inter Society Conference on Thermal Phenomena, pp. 149-156, 2000
[7]. P. Sathyamurthy, P.W. Runstadler, S. Lee, "Numerical and Experimental Evaluation of Planar and Staggered Heat Sinks," Inter Society Conference on Thermal Phenomena, pp. 132-139, 1996.

[8]. A. Dvinsky, A. Bar-Cohen, M.Strelets, "Thermofluid Analysis of Staggered and Inline Pin Fin Heat Sinks," Inter Society Conference on Thermal Phenomena, pp. 157-164, 2000.

[9]. Alpha Company Ltd, http://www.micforg.com.

[10]. Roy W. Knight, Donald J. Hall, John S. Goodling, Richard C. Jaeger, "Heat Sink Optimization with Application to Microchannels," IEEE Transactions on Components, Hybrids, and Manufacturing Technology, Vol 15, pp.832-842, 1992.

[11]. Michael B. Kleiner, Stefan A. Kühn, Karl Haberger, "High Performance Forced Air Cooling Scheme Employing Microchannel Heat Exchangers," IEEE Transactions on Components, Packaging, and Manufacturing Technology-Part A. Vol 18, pp.795-804, 1995.

[12]. Mike Turner, "All You Need To Know About Fans," Electronics Cooling Magazine, May 1996, Vol.2, No 2.

[13]. Robert E. Simons, Roger R. Schmidt, "A simple Method To Estimate Heat Sink Airflow By Pass," Electronics Cooling Magazine, May 1997, Vol.3, No 2. 